\title{
NIR Analysis of Rice Bran Depending on Different Percentages of Rice Polishing
}

\author{
Masanori KUMAGAl*, Toru TAKAHASH", Hitoshi TAKAHASHI*, \\ Nobuaki OGAWA* and Kazuki TOEDA* \\ *Akita Research Institute of Food and Brewing, \\ Arayamachi, Akita 010-1623, Japan \\ **Faculty of Engineering and Resource Science, Akita University, \\ Tegata Gakuencho, Akita 010-8502, Japan \\ E-mail :kumagai@arif.pref.akita.jp
}

\begin{abstract}
Generally, in the case of agricultural products, some difficulties, resulting from the broad peak intensity and extensive overlapping of NIR absorption bands derived from complex chemical components that exist in the sample, arise in relation to specific functionalities of those spectra. This study is intended to specify the NIR spectra of rice bran depending on different percentages of rice polishing. Sample sets were prepared by polishing and grinding away 5\% increments of the original mass of grains to leave $95 \%-45 \%$ of the kernels in successive sets. These samples showed a stepwise increase or decrease of localized chemical constituents: moisture, protein, carbohydrates, etc. Using this approach on a rice bran sample set, basic vibrational information was elucidated for the assignment of absorption bands of rice bran.
\end{abstract}

Key Words : Near infrared spectroscopy, Assignment of absorption bands, Rice bran

\section{Introduction}

Near infrared (NIR) absorption bands are produced when NIR radiation at specific frequencies vibrates at the same frequency as a molecular bond in a sample. However, in the case of agricultural products, some difficulties arise in relation to specific functionalities of those spectra. Those difficulties stem from the broad peak intensity and extensive overlapping of NIR absorption bands derived from complex chemical components that exist in the sample.

Therefore, it is often the case that the empirical models are used for the calibration models to relate the concentration of some analyte found in a sample to the NIR spectral data collected from that sample using a variety of multivariate analysis or some chemometrical techniques. No one would deny that the statistical treatment has contributed to the tremendous expansion of popularity of the NIR spectroscopy. But it is often claimed that such approaches can result in the production of a "black box" technique, in that very little is understood about the assignment of the observed bands or about the rules of the vibrational spectroscopy underlying a NIR spectrum.

This study is intended to specify the NIR spectra and elucidate basic vibrational information for the assignment of absorption bands of rice bran.

\section{Experimental}

\subsection{Materials}

Rice samples were collected from the sake- brewing department of the Akita Research Institute of Food and Brewing. The investigated rice samples consisted of Akita Sake Komachi, which is suitable for sake brewing. Sample sets were prepared by polishing and grinding away $5 \%$ increments of the original grain mass to leave $95 \%-45 \%$ of the kernels in successive sets.

\subsection{NIR spectroscopy and data analysis}

The NIR diffuse reflection spectra in the $1100-2500 \mathrm{~nm}$ wavelength regions at $2 \mathrm{~nm}$ intervals were recorded on a near infrared spectrophotometer (NIRS 6500; Foss-NIRSystems Inc.) fitted with a sample transport module. Each sample was packed into a circular sample holder in which it was compressed against a quartz window. The NIR spectra were recorded three times for each sample. Data analyses were performed using a software program (Pirouette ${ }^{\circledR}$; Infometrix, Inc.).

\subsection{Reference analysis}

Reference laboratory values of moisture, protein, fat, ash, and carbohydrates were determined in conformity with Standard Tables of Food Composition in Japan. Analyses were repeated twice for each sample.

\section{Results and Discussion}

\subsection{Properties of the sample sets}

Table 1 shows the investigated sample sets and characterization of their properties. These samples showed a stepwise increase or decrease of localized chemical constituents depending on the degree of grinding treatment. 
Table 1 Properties of the sample sets.

\begin{tabular}{cccccc}
\hline Increments & $\begin{array}{c}\text { Moisture } \\
\%\end{array}$ & $\begin{array}{c}\text { Protein } \\
\%\end{array}$ & $\begin{array}{c}\text { Fat } \\
\%\end{array}$ & $\begin{array}{c}\text { Ash } \\
\%\end{array}$ & $\begin{array}{c}\text { Carbohydrate } \\
\%\end{array}$ \\
\hline raw rice & 12.68 & 5.88 & 1.41 & 1.34 & 78.68 \\
5 & 11.08 & 9.90 & 10.04 & 7.43 & 61.55 \\
10 & 11.17 & 13.83 & 8.54 & 7.20 & 59.26 \\
15 & 10.79 & 14.58 & 5.83 & 4.78 & 64.02 \\
20 & 10.34 & 13.17 & 3.41 & 2.95 & 70.13 \\
25 & 9.86 & 11.62 & 2.35 & 1.75 & 74.41 \\
30 & 9.68 & 9.73 & 1.40 & 1.03 & 78.16 \\
35 & 9.79 & 8.16 & 1.14 & 0.67 & 80.24 \\
40 & 9.04 & 7.04 & 0.97 & 0.44 & 83.51 \\
45 & 8.76 & 6.43 & 0.48 & 0.45 & 83.87 \\
50 & 8.24 & 5.62 & 0.55 & 0.35 & 87.24 \\
55 & 7.26 & 4.76 & 0.53 & 0.33 & 87.12 \\
60 & 6.99 & 4.28 & 0.52 & 0.30 & 88.17 \\
$45 \%$ of kernel & 8.67 & 2.78 & 0.26 & 0.11 & 8 \\
\hline
\end{tabular}

\subsection{NIR spectra}

Figure 1 shows the original NIR spectra of sample sets. The relationship between the sample properties and absorbance of original spectra is illustrated in Figure 2. The spectra might contain some variance arising from scattering and spreading, which gradually increase with the wavelength number. These observations account for the differences of the samples' powder surfaces in particle size, orientation, etc., which induce variations in diffuse reflection spectra.

Consequently, to remove extraneous systematic variations from nonscattering origins, standardized normalization treatment was applied for the original NIR spectra $[1,2]$. Figure 3 shows NIR spectra after standardized treatment. The relationship between the sample properties and absorbance of standardized spectra is illus-

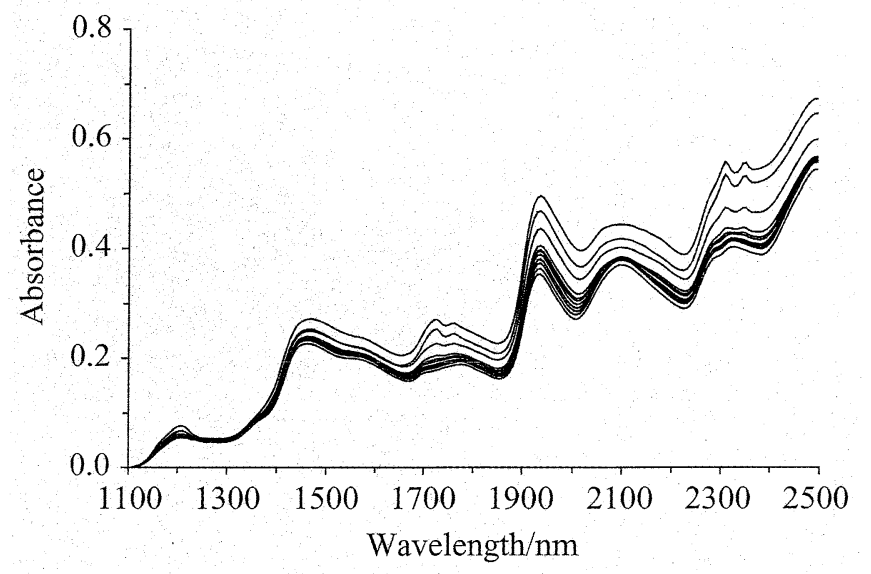

Figure 1 Original near infrared spectrà of sample sets.

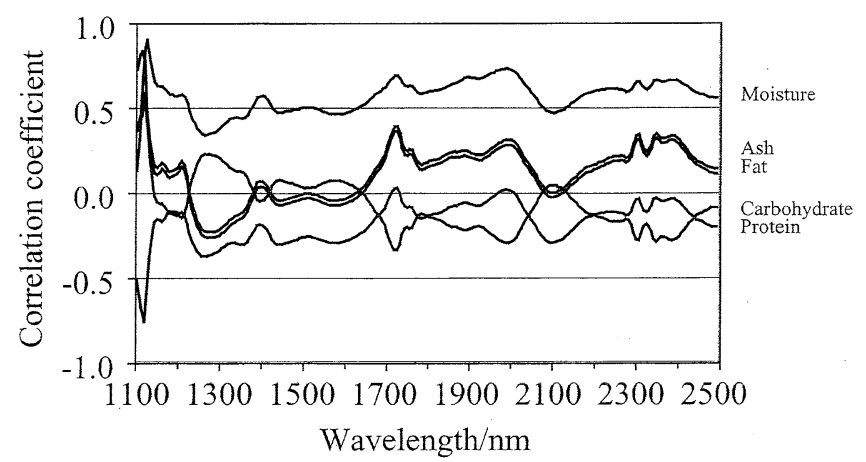

Figure 2 The relationship between the sample properties and absorbance of original spectra. trated in Figure 4. Only narrower data spreads than those of the original spectra are represented by component differences of the investigated samples. Figure 4 depicts a characteristic assignment of each structural chemical component, in contrast to Figure 2.

In Figure 4, moisture was strongly correlated in the region of $1906-2026 \mathrm{~nm}$, suggesting the O-H stretch first overtone (1908 $\mathrm{nm}$ ) and the O-H bend second overtone (1940 nm) [3]. Similarly, carbohydrate was correlated strongly with the O-H bend/C-O stretch combination $(2104 \mathrm{~nm})$, suggesting a starch structure. Protein was correlated in the region of 1432-1648, suggesting the $\mathrm{N}-\mathrm{H}$ stretch first overtone [3]. However, absorbance at $2180 \mathrm{~nm}$ related to the combination band of protein showed no clear correlation. The absorption bands at $2074 \mathrm{~nm}, 2308 \mathrm{~nm}$ and 2392 $\mathrm{nm}$, which are assigned to oil vibration [3], were closely related

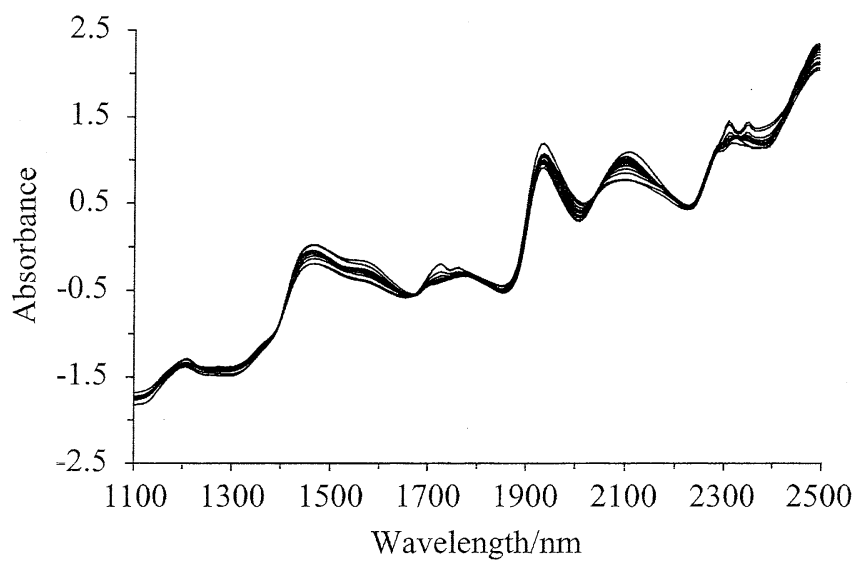

Figure 3 Near infrared spectra after the standardized treatment.

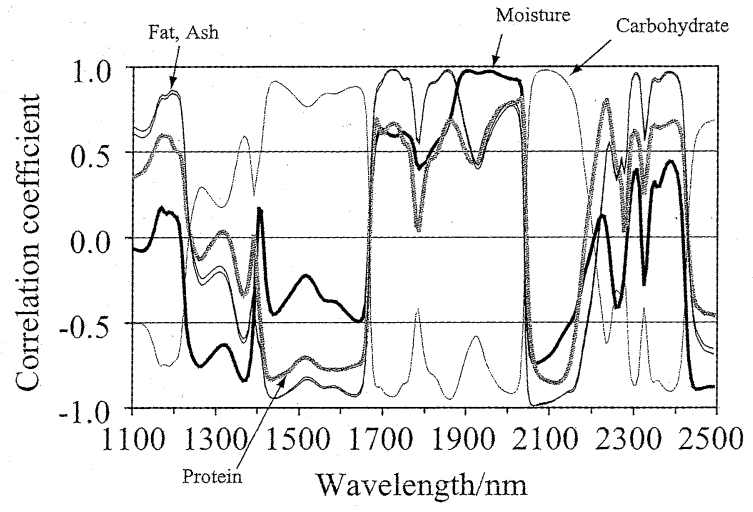

Figure 4 The relationship between the sample properties and absorbance of standardized spectra. 

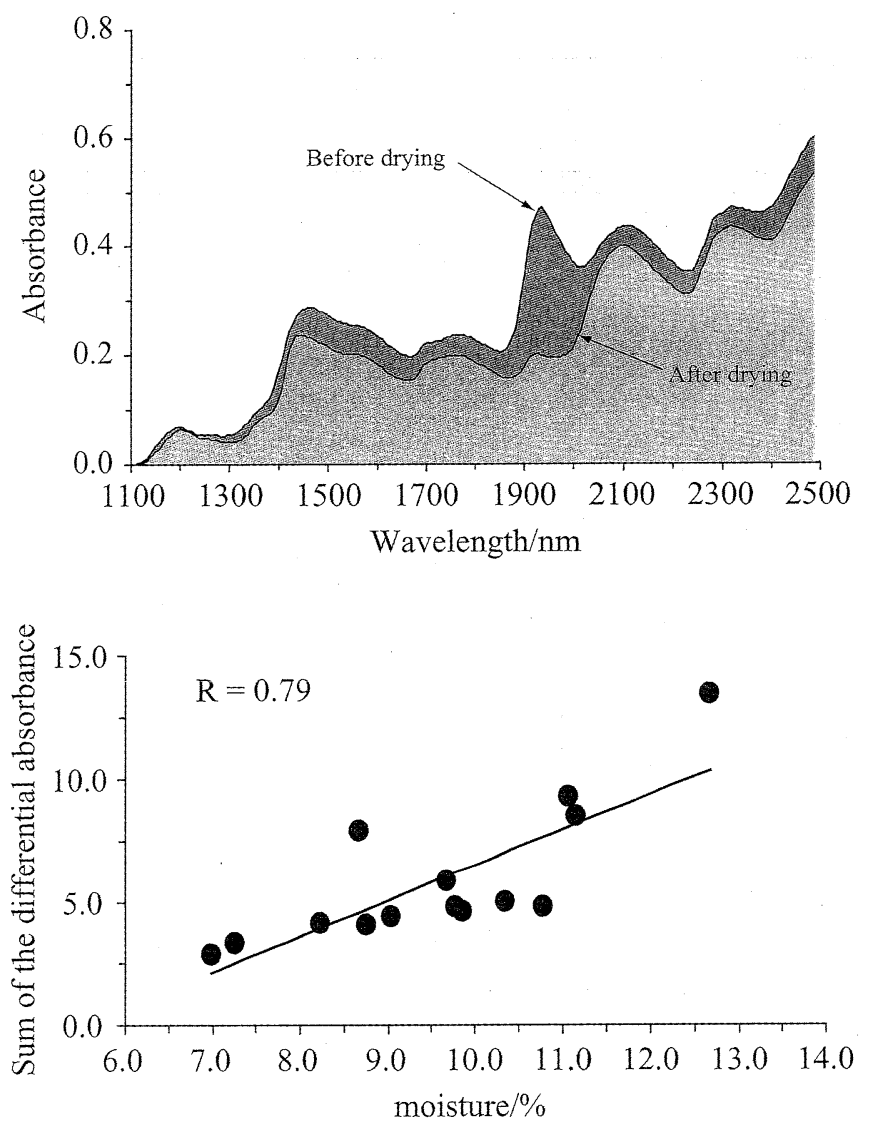

Figure 5 Original spectra of before-and-after drying (upper), and relationship between moisture and sum of differential absorbance (lower).
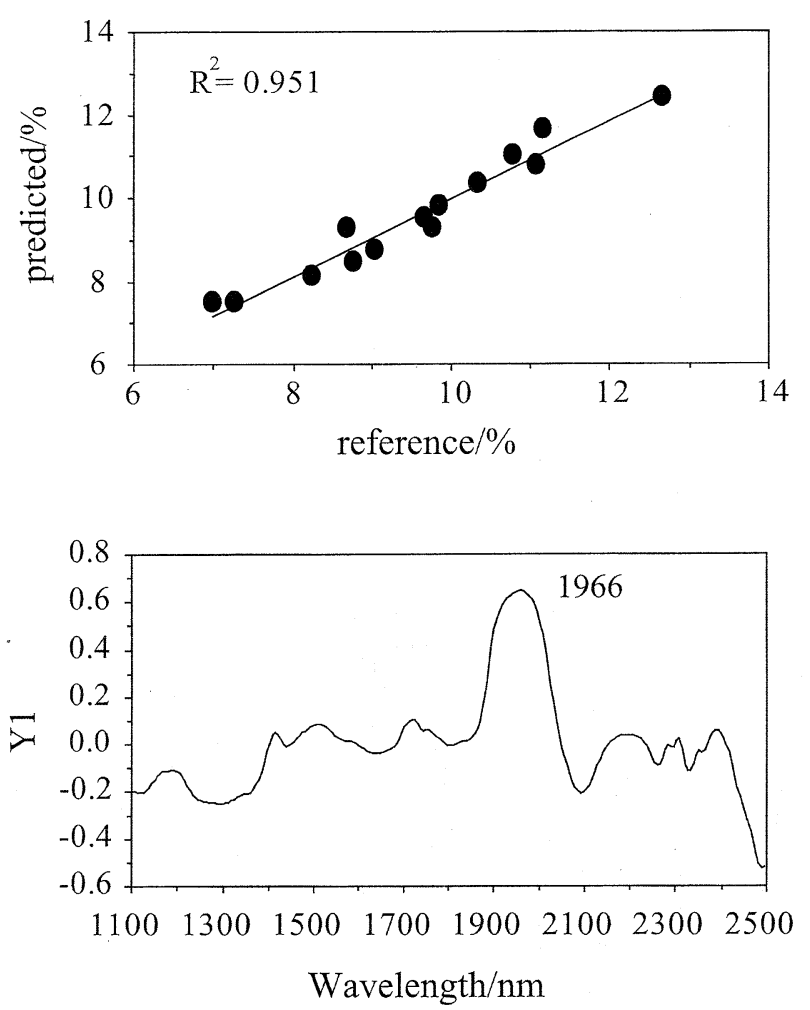

Figure 6 Relationship between predicted and reference values (upper), and regression vector (lower) for moisture.
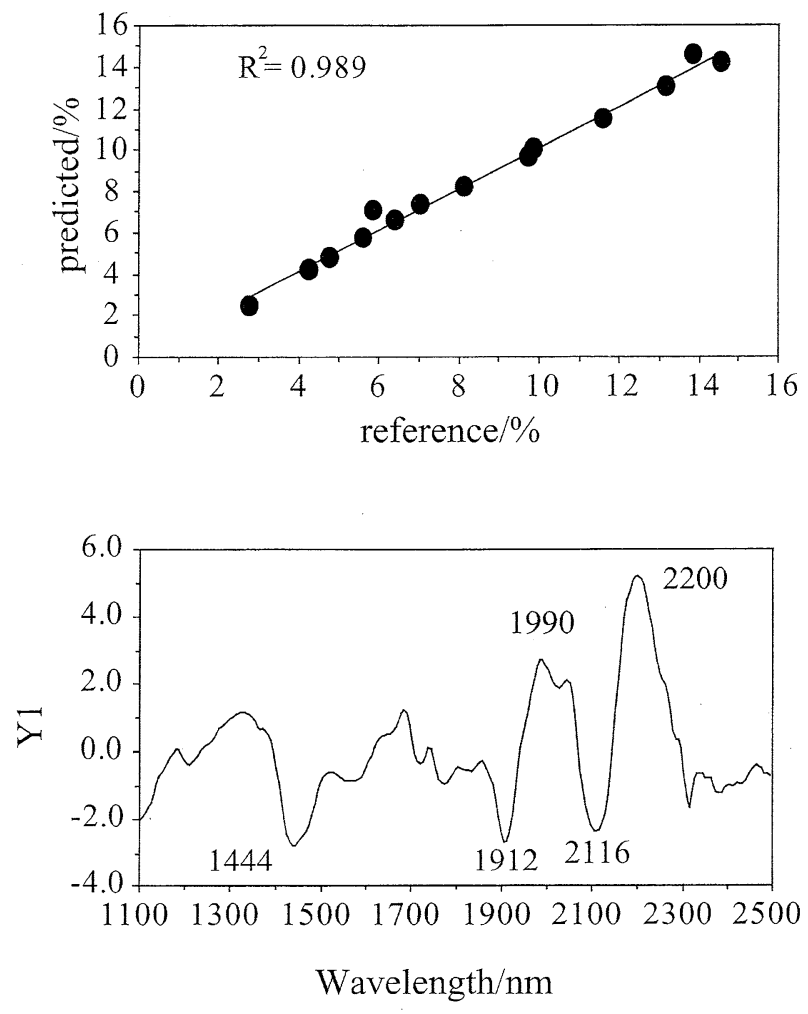

Figure 7 Relationship between predicted and reference values (upper), and regression vector (lower) for protein.
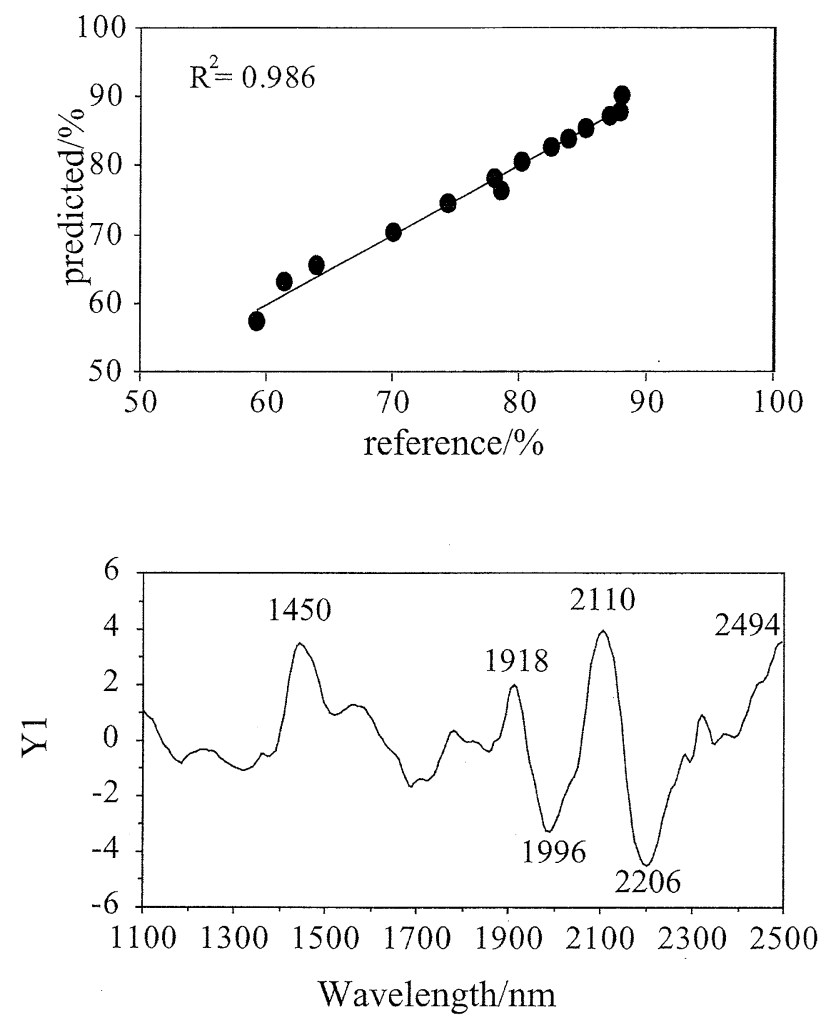

Figure 8 Relationship between predicted and reference values (upper), and regression vector (lower) for carbohydrate. 
with fat content. It is noteworthy that the correlation coefficients of fat were very similar to those of ash. The reason why both components were indistinguishable using NIR spectral changes might be the strong internal correlation between fat and ash.

The sum of differential absorbance, subtracting the original NIR spectra after drying treatment $\left(135^{\circ} \mathrm{C}, 1 \mathrm{~h}\right)$ from those before drying (Figure 5, upper), was in accord with the moisture content of the samples (Figure 5, lower). The largest gap of differential absorbance at $1940 \mathrm{~nm}$ appeared to be assigned to the $\mathrm{O}-\mathrm{H}$ bend second overtone [3].

\subsection{Predictive performance}

The predictive performances for moisture, protein and carbohydrate are shown respectively in Figures $6-8$. Those for fat and ash were omitted because of their strong internal correlation. The performance of the predictive partial least squares (PLS) model were tested by estimating the standard errors of prediction (SEP) on a set of independent samples for each constituent.

Figure 6 portrays SEP as 0.256 and the slope as 0.971 . The most effective wavelength for moisture prediction was $1966 \mathrm{~nm}$, suggesting the $\mathrm{O}-\mathrm{H}$ stretch/O-H bend combination [3] .

Figure 7 shows SEP of 0.170 and a slope of 0.998 . The important wavelengths for the prediction model were $1444 \mathrm{~nm}(\mathrm{CH}$ combination), $1912 \mathrm{~nm}$ ( $\mathrm{C}=\mathrm{O}$ stretch second overtone $), 1990 \mathrm{~nm}$ (N-H combination), and $2200 \mathrm{~nm}$ ( $\mathrm{N}-\mathrm{H}$ bend second overtone), etc. [3]. The wavelength at $2116 \mathrm{~nm}$ was not assigned by the literature as indicating a wavelength of bond absorption.

Finally, Figure 8 illustrates SEP of 0.448 and a slope of 0.998. The important wavelengths for the predictive model were $1450 \mathrm{~nm}$ (O-H stretch first overtone), $1918 \mathrm{~nm}$ (O-H stretch first overtone), 1996 and $2110 \mathrm{~nm}$ (O-H, C-O combination), $2206 \mathrm{~nm}$ (C-H, C-O combination), and $2494 \mathrm{~nm}$ (C-H, C-C combination), etc. These are all associated with a starch structure [3].

\section{Conclusions}

This study specified the NIR spectra of rice bran depending on different percentages of rice polishing. Sample sets were prepared by polishing and grinding away $5 \%$ increments of the original mass of grains to leave $95 \%-45 \%$ of the kernels in successive sets. These samples showed a stepwise increase or decrease of localized chemical constituents: moisture, protein, carbohydrates, etc. Using this approach on a rice bran sample set, it was elucidated basic vibrational information for assignment of absorption bands of rice bran. The chemistry-based interpretation of NIR spectra is the most essential and important concept for development of NIR spectroscopy.

\section{References}

[1] Kumagai, M., Karube, K., Sato, T., Ohisa, N., Amano, T., Kikuchi, R., and Ogawa, N., "A Near-Infrared Spectroscopic Discrimination of Noodle Flours using a PrincipalComponent Analysis coupled with Chemical Information," Anal. Sci., Vol.18, pp.1145-1150, (2002).

[2] Kumagai, M., Matsuura, N., Ohisa, N., Amano, T. and Ogawa, N., "Application of a Portable Near Infrared Spectrometer for the Manufacturing Process of Noodle Products," J. Near Infrared Spectrosc., Vol.12, pp.127-131, (2004).

[3] Burns, D. A. and Ciurczak, E. W., "Handbook of NearInfrared Analysis," Marcel Dekker, New York, (2001). 